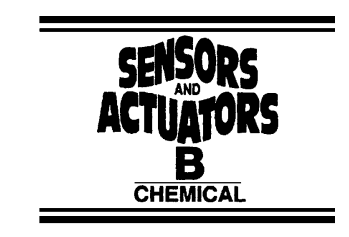

\title{
An array-based CMOS biochip for electrical detection of DNA with multilayer self-assembly gold nanoparticles
}

\author{
Yi-Ting Cheng, Ching-Chin Pun, Chien-Ying Tsai, Ping-Hei Chen* \\ Department of Mechanical Engineering, National Taiwan University, No. 1, Sec. 4, Roosevelt Road, Taipei 10617, Taiwan, ROC
}

Received 27 July 2004; received in revised form 25 November 2004; accepted 29 December 2004

Available online 4 February 2005

\begin{abstract}
This paper presents an array-based CMOS biochip for DNA detection using self-assembly multilayer gold nanoparticles (AuNPs). The biochip is fabricated by a TSMC $0.35 \mu \mathrm{m}$ standard CMOS process and post-CMOS micromachining processes. Before taking DNA detection measurements, self-assembly monolayer of AuNPs is established on $\mathrm{SiO}_{2}$ surface between two microelectrodes. The gap distance between the two microelectrodes in this study is less than $800 \mathrm{~nm}$. First, capture oligonucleotide strands are immobilized on the top surface of self-assembly monolayer of AuNPs. On each measuring spot, selective binding occurs among thiol-modified probe oligonucleotide strands, capture oligonucleotide strands, and target oligonucleotide strands if the target oligonucleotide strands are complementary with the probe and capture oligonucleotide strands in the sample solution. Finally, through a self-assembly process between suspended gold nanoparticles and the thiol-modified end of probe oligonucleotide strand, a multilayer of AuNPs on the nanogap surface can be established. The DNA hybridization can be determined by a sharp decrease in impedance over the nanogap between the two microelectrodes, which results from an increase in the number of AuNPs layers. Measured results show that the electric current of multilayer AuNPs is about 1000 times greater than that of monolayer AuNPs. The effect of nanogap distance is also investigated. It is shown that the electrical conductivity decreases with the increase of gap distance.
\end{abstract}

(C) 2005 Elsevier B.V. All rights reserved.

Keywords: CMOS biochip; DNA identification; Gold nanoparticles; Self-assembly

\section{Introduction}

Recent progress in clinical genetic research proves that detections of genetic diseases and viral infections can be done by using a DNA microarray chip to identify specific DNA sequences from fluid samples of a patient. However, some serious problems arise in conjunction to the use of DNA microarray for identification of DNA. First of all, the number of specific DNA sequences in a sample might not be enough for precise detection. To solve the problem, DNA in a sample needs to be duplicated through a polymerase chain reaction (PCR) that hinders a fast diagnosis. The second problem is that the fluorescence detection system for DNA identification used in microarray is large and expensive. Meanwhile,

\footnotetext{
* Corresponding author. Tel.: +8862 33662689; fax: +886223670781.

E-mail address: phchen@ntu.edu.tw (P.-H. Chen).
}

precision scanning devices and professional technicians are also needed. Thirdly, the yield rate of microarray fabricated by an MEMS process is, at present, still too low for mass production.

Taton et al. [1-3] demonstrated a DNA detection approach by utilizing UV absorption of multilayered gold nanoparticles on a glass substrate. The detected signal can be amplified by augmentation of gold nanoparticles through a chemical reduction of silver in the sample solution. The sensitivity of this detection method exceeds that of conventional fluorescence detection method by two orders of magnitude in DNA identification. Another novel DNA detection method was reported by the same research group in 2002 [4]. This method utilizes the binding of oligonucleotides functionalized gold nanoparticles in a $20 \mu \mathrm{m}$ gap between two electrodes to vary impedance of the gap, and the use of silver enhanced solution is essential to amplify the originally unde- 
tectable signal. In 2002, $\mathrm{Li}$ and co-workers [5] reported the electrical behavior of self-assembly gold nanoparticle layers using 1,6-hexanedithiol as crosslinkers. In 2004, Tsai et al. [6] presented an electrical detection method that uses multiple layers of self-assembly gold nanoparticles for signal amplification between nanogap electrodes instead of silver enhancement. Recently, some DNA analysis methods combined with CMOS technology were published [7-12].

In this paper, an electrical detection method is employed in an array-based biochip for DNA identification. The biochip is fabricated by standard CMOS technology combining post-CMOS micromachining processes. The electrical detection method utilizing self-assembly gold nanoparticle multilayer without silver enhancement is used to detect the hybridizations between probe, capture, and target oligonucleotides. With proper circuit design for current amplification on the present array-based biochip, a simple and inexpensive current meter can be used for DNA detection process.

\section{Operating principle of the DNA biochip}

The sequence-selective DNA detection by an array-based CMOS biochip relies on a specific hybridization of surfacebound, single-strand capture oligonucleotides and suspended probe oligonucleotides with their complementary target oligonucleotides in the sample solution. In order to obtain a measurable current across electrodes after DNA hybridization, multilayer gold nanoparticle structure over the gap between electrodes is used in this work rather than monolayer with silver enhancement [4]. The procedure of the establishment of multilayer gold nanoparticles, as shown in Fig. 1, can be stated as follows. Firstly, a self-assembly gold nanoparticle monolayer is immobilized in the gap by carbon strains after a nanogap is fabricated between two microelectrodes. Carbon strains help to attach gold nanoparticles to the $\mathrm{SiO}_{2}$ surface. Then, the single strand thiol-modified capture oligonucleotides (labeled as cDNA in Fig. 1) is added to the nanogap and immobilized onto the surface of monolayer gold nanoparticles. Selective binding occurs among immobilized capture oligonucleotide strand, suspended probe oligonucleotide (labeled as pDNA in Fig. 1), and target oligonucleotide strand (labeled as tDNA in Fig. 1) in the sample solution. One end of the probe oligonucleotide DNA is modified by thiol. Finally, the measured spot is rinsed with a PBS solution before adding gold nanoparticles. If there is no hybridization among the oligonucleotide strands, all the probe oligonucleotide and target oligonucleotide will be washed away. No gold nanoparticles would be attached to the capture oligonucleotide immobilized on the gold nanoparticle monolayer. However, if complementary hybridization occurs between oligonucleotide strands, gold nanoparticle probes can be attached to the thiol-modified probe oligonucleotide to form multilayer of gold nanoparticles for electrical detection. Sequences of the oligonucleotides are shown in Table 1.
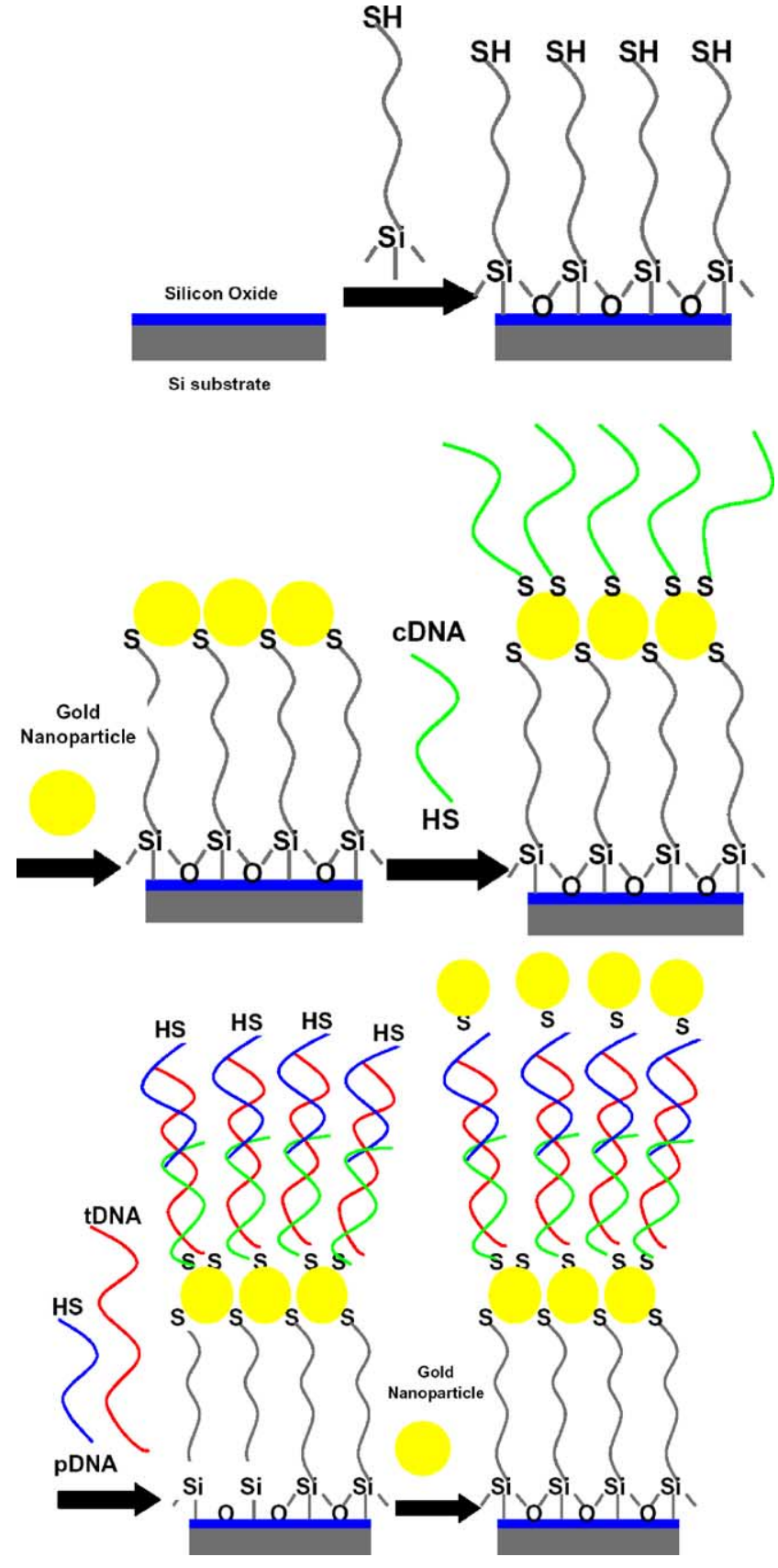

Fig. 1. Steps for establishing multilayer of self-assembly gold nanoparticles.

The filling of gold nanoparticle probes between the microelectrodes allows electric current to pass through. Hence, the conductivity between the two microelectrodes will be significantly improved when DNA hybridization causes the

Table 1

Sequences of capture, probe, and target oligonucleotide strands

\begin{tabular}{ll}
\hline Name & Sequence \\
\hline cDNA & $3^{\prime}$-SH-A10CCTAATAACAAT-5' \\
pDNA & $3^{\prime}$-TTATAACTATTCCTAA10-SH-5' \\
tDNA & $5^{\prime}$-GGATTATTGTTAAATATTGATAAGGAT-3'
\end{tabular}


gold nanoparticle structure to transform from a monolayer to a multilayer. The conductivity measurement through microelectrodes can be made to identify whether hybridization of oligonucleotide strands occurs or not. The use of gold nanoparticle multilayer presented here is to increase the density of gold nanoparticle immobilization over the gap between microelectrodes, which can amplify the electrical signal without using complicated means of silver enhancement.

\section{Post-CMOS processing of the DNA biochip}

The DNA biochip is fabricated by TSMC $0.35 \mu \mathrm{m}$ 1P4M standard CMOS process and it consists of several components, including microelectrodes with nanogap for electrical detection, heating elements, and temperature sensors for checking thermal stringency of DNA hybridization. The rationale for a temperature control system can be explained as follows.

Both the specificity and selectivity of microarray depend on clear variation in dissociation properties between complementary hybridization and mismatch hybridization. However, the temperature difference of thermal stringency to denature binding oligonucleotides between complementary oligonucleotides and mismatch ones is not very distinctive. Thus, a precise temperature control mechanism is needed on the biochip for thermal stringency check.

In addition to nanogap microelectrodes, the array-based CMOS biochip contains thermopile-based temperature sensor and heating elements. The embedded temperature sensor in the array-based CMOS biochip is used to make spot measurement of temperature on the nanogap between microelectrodes. An effective temperature feedback control system, which is external to the chip, is connected to a polysilicon heater. The CMOS thermopile structure is formed by $\mathrm{Al} /$ polysilicon layers and consists of 39 thermocouples. In order to eliminate the heat loss through the silicon substrate during measurement, thermal isolation structures are obtained by using front-end bulk etching in TMAH etching solution.

There are three etching steps to obtain microelectrodes with nanogap and temperature sensor structures on the chip simultaneously. The etching processes as a whole are realized by the concept of maskless etching.

Fig. 2 shows the complete post-CMOS micromachining process flow. The metal structures in the etching holes, without protection from passivation layer, will be removed by wet etching because the passivation layer is open on the top of the etching holes. For the current design of array-based CMOS biochip, there are two types of etching holes: one is used for removing silicon substrate to increase the sensitivity of thermopile; another for etching the metal layer on the top of microelectrodes to decrease the time of dry etching.

The first step is to remove metal layers with traditional Piranha solution at $80^{\circ} \mathrm{C}$ for $30 \mathrm{~min}$, a 3:1 mixture of concen-

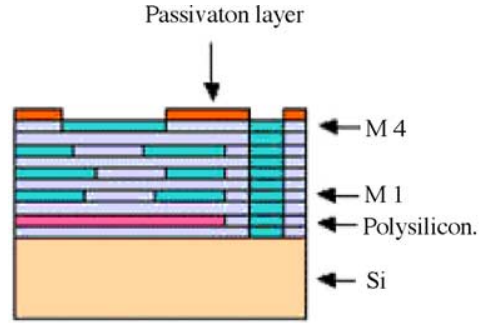

(a)

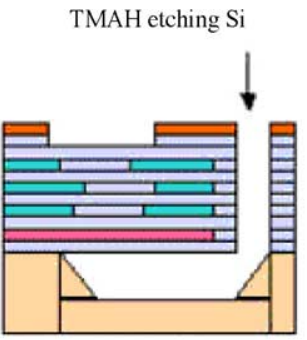

(c)

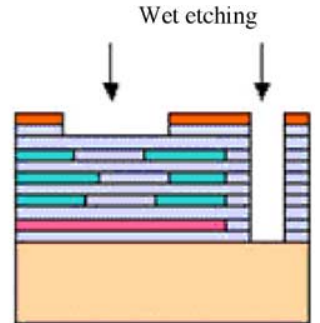

(b)

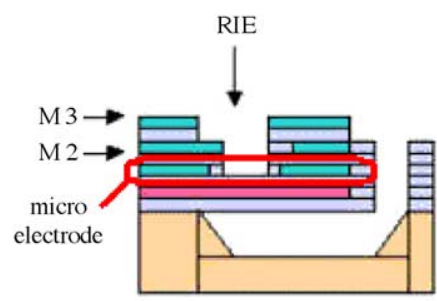

(d)
Fig. 2. Post-CMOS micromachining process flow: (a) cross-sectional view of CMOS biochip; (b) wet etching; (c) TMAH etching and (d) RIE dry etching.

trated sulfuric acid $\left(\mathrm{H}_{2} \mathrm{SO}_{4}\right)$ with hydrogen peroxide $\left(\mathrm{H}_{2} \mathrm{O}_{2}\right)$. The isotropic wet etching solution flows and etches along the etching holes that are originally filled with metal structures. Then, the front-end bulk etching anisotropic wet etching is applied to remove silicon substrate as some parts of silicon substrate are exposed after the Piranha etching. The anisotropic wet etching solution used for silicon is $8 \%$ TMAH solution at $90^{\circ} \mathrm{C}$, which is compatible with CMOS process.

The final step is to employ an anisotropic dry etching-reaction ion etching (RIE, model: RIE-10N, SAMCO) to remove $\mathrm{SiO}_{2}$ with $\mathrm{CF}_{4}: 20 \mathrm{sccm}, \mathrm{O}_{2}: 5.2 \mathrm{sccm}$, pressure: $7 \mathrm{~Pa}$, and rf power: $100 \mathrm{~W}$ for $125 \mathrm{~min}$.

Since dry etching gas exhibits high selectivity between aluminum and $\mathrm{SiO}_{2}$, the metal layers can be used as etching mask. Due to anisotropic etching of $\mathrm{SiO}_{2}$ by RIE, the pattern of microelectrodes defined by the layers of metal 2 and metal 3 is formed. Microelectrodes lie on the layer of metal 1 . The reaction ion etching continues until the microelectrodes appear. The surface of the nanogap between microelectrodes is still made of $\mathrm{SiO}_{2}$ for DNA immobilization. Fig. 3(a) and (b) illustrates the micrograph of the array-based CMOS biochip before and after post-micromachining process, respectively. In Fig. 3(b), the etching hole for TMAH etch has been removed. The biochip has a dimension of $2 \mathrm{~mm} \times 2 \mathrm{~mm}$ and there are 40 measuring spots on a biochip. Fig. 4 shows a field-emission scanning electron microscopic (FE-SEM: HITACHI, S-4000) picture of microelectrodes with nanogap after post-CMOS micromachining process. The nanogap is $350 \mathrm{~nm}$ in width, $5 \mu \mathrm{m}$ in length, and $3 \mu \mathrm{m}$ in thickness. 


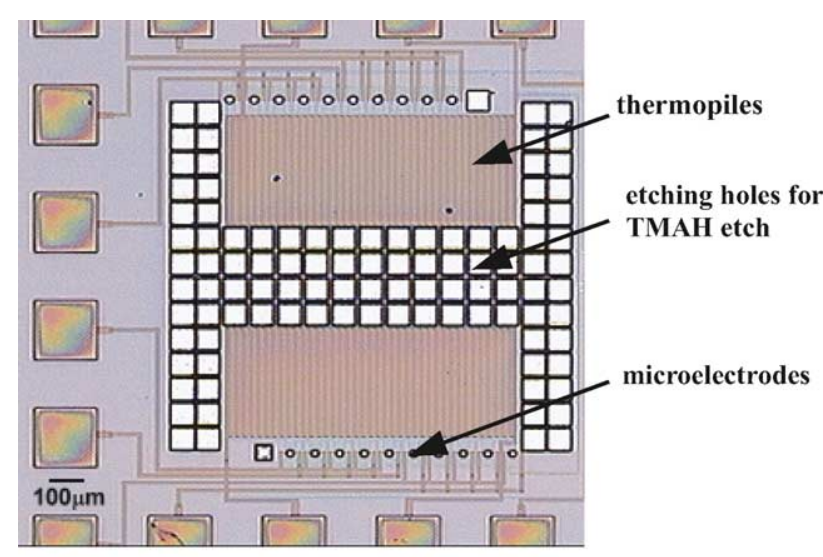

(a)

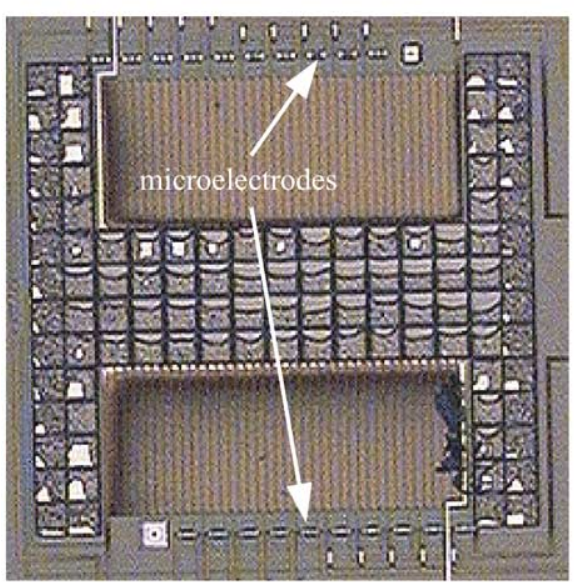

(b)

Fig. 3. Micrograph of the array-based CMOS biochip (a) before postmicromachining processes and (b) after post-micromachining processes.

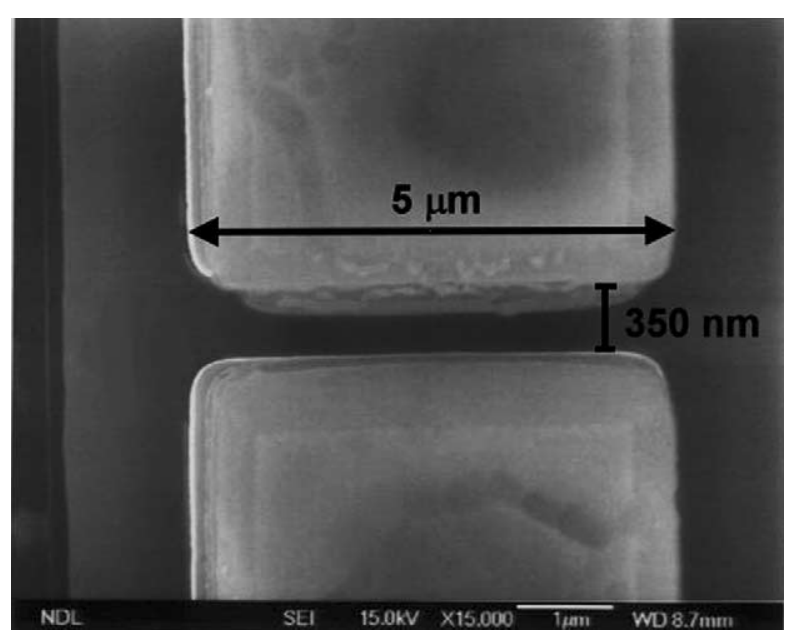

Fig. 4. FE-SEM picture of microelectrodes with nanogap after post-CMOS micromachining processes.

\section{Experiments}

\subsection{Preparation of Au nanoparticles (AuNPs)}

Gold nanoparticles (AuNPs) are prepared by the reduction of aqueous hydrogen tetrachloroaurate $\left(\mathrm{HAuCl}_{4}\right.$, Aldrich Chem. Co.) with trisodium citrate and tannic acid (Aldrich Chem. Co.) using a standard procedure [13]. A solution of $\mathrm{HAuCl}_{4}(1 \mathrm{mg})$ in distilled water $(80 \mathrm{ml})$, and another solution of trisodium citrate $(0.05 \mathrm{mg})$ and tannic acid $(0.01 \mathrm{mg})$ in distilled water $(20 \mathrm{ml})$ are prepared. Both solutions are heated to $60^{\circ} \mathrm{C}$ and are subsequently mixed. After the development of a crimson color, the aqueous solution is heated to boiling for $10 \mathrm{~min}$. High-resolution transmission electronic micrograph (HR-TEM, model: H-7000, Hitachi) is used to determine the size of AuNPs. The resulting AuNP solution has a strong surface plasma resonance at $523 \mathrm{~nm}$ and the size of the AuNPs is found to be in the range of $12 \pm 4 \mathrm{~nm}$.

\subsection{Procedures for DNA hybridization of self-assembly multilayer Au nanoparticles}

\subsubsection{Silanization of substrate}

The substrate is cleaned by immersion in 1:1 concentrated $\mathrm{HCl}: \mathrm{MeOH}$ for $30 \mathrm{~min}$, followed by rinsing with deionized $\mathrm{H}_{2} \mathrm{O}$. The substrate is then immersed in concentrated $\mathrm{H}_{2} \mathrm{SO}_{4}$ for $5 \mathrm{~min}$ and is rinsed with DI water. 3Mercaptopropyltrimethoxysilane (MTPMS) is prepared as $1 \mathrm{mM}$ solution in DMSO. After being rinsed with DMSO and dried under $\mathrm{N}_{2}$, the substrate is placed in the silane solution for at least $2 \mathrm{~h}$ at room temperature.

\subsubsection{Establishing monolayer AuNPs on silanized substrate}

The functionalized substrate is immersed in the gold nanoparticles $(12 \mathrm{~nm}$ diameter) solution for $8-12 \mathrm{~h}$, then rinsed with distilled water and dried under $\mathrm{N}_{2}$.

\subsubsection{Immobilization of thiolated capture \\ oligonucleotide strands onto the top surface of \\ monolayer of AuNPs}

Capture oligonucleotide strands are immobilized onto the top surface of monolayer of AuNPs by spotting a $10 \mathrm{mM}$ HEPES/5 mM EDTA buffer solution of the appropriate alkylthiol-modified oligonucleotide $(0.1 \mu \mathrm{M})$ on the chip by manual pipetting. After spotting capture oligonucleotides, the chip is stored in a humidity chamber for $24 \mathrm{~h}$ to allow the coupling reaction between gold nanoparticles and alkylthiol-modified capture DNA to take place. Finally, the DNA-functionalized chip is washed with ethanol and water and then blown dry in $\mathrm{N}_{2}$.

\subsubsection{DNA hybridization and the formation of second layer of gold nanoparticles}

To perform measurement, the chip is immersed into a $10 \mathrm{mM}$ HEPES/5 mM EDTA buffer solution of target DNA 
( $1 \mathrm{nM}$ ) for $4 \mathrm{~h}$ and is rinsed with a $0.3 \mathrm{M}$ PBS solution. It is then treated with a $10 \mathrm{mM}$ HEPES/5 mM EDTA buffer solution of probe DNA $(0.1 \mu \mathrm{M})$ for another $4 \mathrm{~h}$ and is rinsed with a $0.3 \mathrm{M}$ PBS solution. Finally, the chip is immersed in a $0.3 \mathrm{M}$ PBS solution of $12 \mathrm{~nm}$ diameter gold particles for $2 \mathrm{~h}$ and is rinsed with a $0.3 \mathrm{M}$ PBS solution again. If hybridization occurs among capture, target and probe oligonucleotide strands, added gold nanoparticles can attach to the thoilated end of probe DNA to form a second layer of AuNPs. If there is no hybridization in the solution, both target and probe DNAs are rinsed away. As a result, added gold nanoparticles cannot attach to the free end (no thiolated modification) of capture oligonucleotide strands to form any second layer of AuNPs.

\section{Results and discussion}

In this study, the change in conductivity of gold nanoparticle structure between two microelectrodes can be measured to determine whether DNA hybridization occurs or not. If complementary hybridization among oligonucleotide strands does occur, a gold nanoparticle multilayer will exist over the

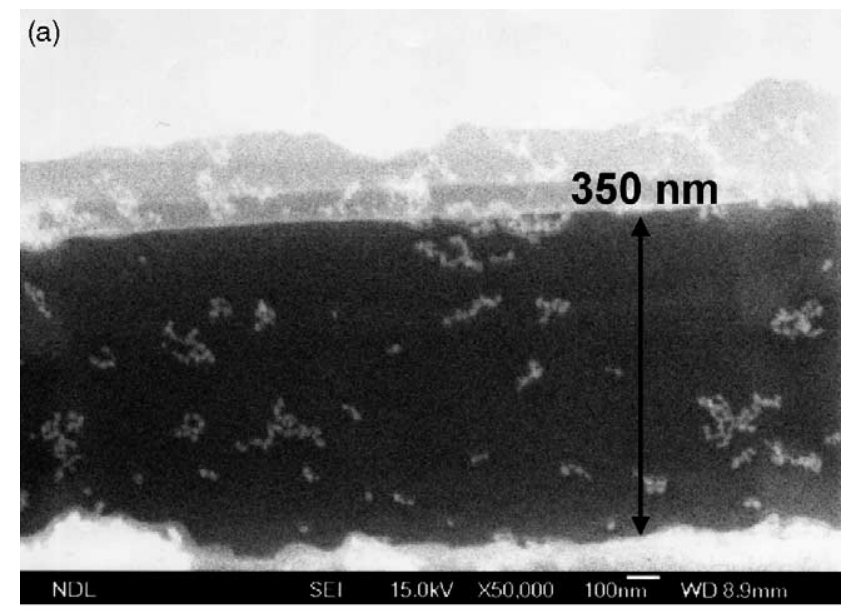

(b)

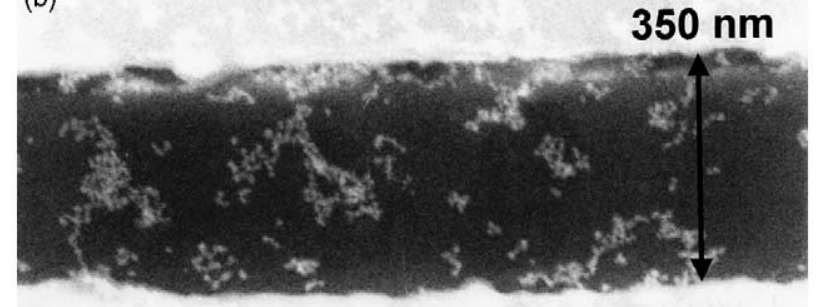

Fig. 5. FE-SEM micrograph of self-assembly layered gold nanoparticles over the nanogap between microelectrodes (a) monolayer and (b) multilayer. nanogap between two microelectrodes. If not, there will only be a monolayer of gold nanoparticle over the nanogap. It is expected that the impedance of gold nanoparticle structure can vary with the number of gold nanoparticle layers.

Fig. 5(a) and (b) respectively shows the FE-SEM micrograph of gold nanoparticle monolayer and multilayer immobilized in the gap between two microelectrodes. The white spots in the micrographs are gold nanoparticles. The distribution density of gold nanoparticles of multilayer is much larger than that of monolayer. It can be observed that the gold nanoparticles are discretely scattered but not continuously distributed on the gap. Fig. 6(a) and (b) show the $I-V$ characteristic curves for monolayer and multilayer of gold nanoparticle structure in $350 \mathrm{~nm}$ gap, respectively. In this study, we use the semiconductor device analysis system (HP 4156) to measure electrical signal. The noise level of this machine is about several fA and the scanning rate for the $I-V$ curve measurement is $10 \mathrm{mV} / \mathrm{s}$. After successful gold immobilization of nanoparticle monolayer in the gap, the electric current passing through the two microelectrodes is lower than $10 \mathrm{pA}$ (Fig. 6(a)) with the applied voltage of $1 \mathrm{~V}$. Although gold nanoparticles are not continuously distributed, the current passing through microelectrodes is still detectable. It is assumed to be the result of the tunneling effect through discrete gold nanoparticles. The resulting $I-V$ curve for the gold nanoparticle monolayer follows the Ohm's law but with some fluctuations. After hybridization among oligonucleotide strands over the gold nanoparticle monolayer, the electric current passing through two microelectrodes for the multilayer of self-assembly gold nanopar-

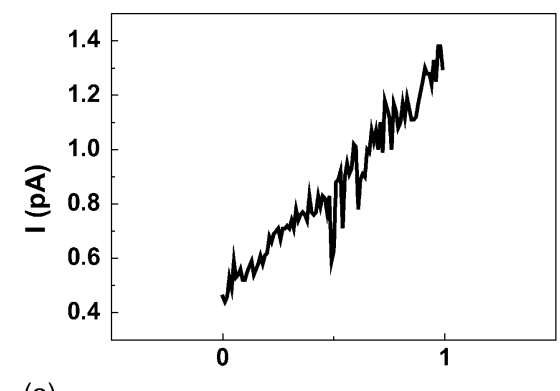

(a)

V (volt)

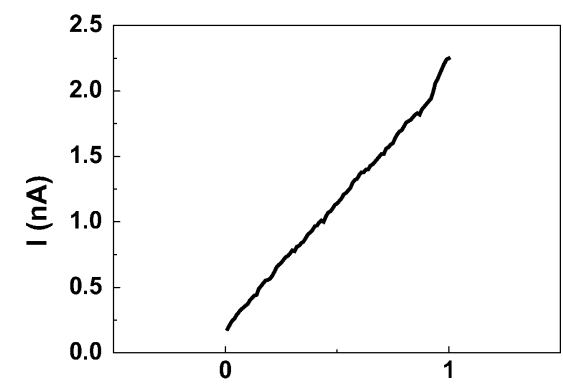

(b)

V (volt)

Fig. 6. $I-V$ characteristic curves of self-assembly layered gold nanoparticles over a $350 \mathrm{~nm}$ nanogap: (a) monolayer and (b) multilayer. The scanning rate is $10 \mathrm{mV} / \mathrm{s}$. 


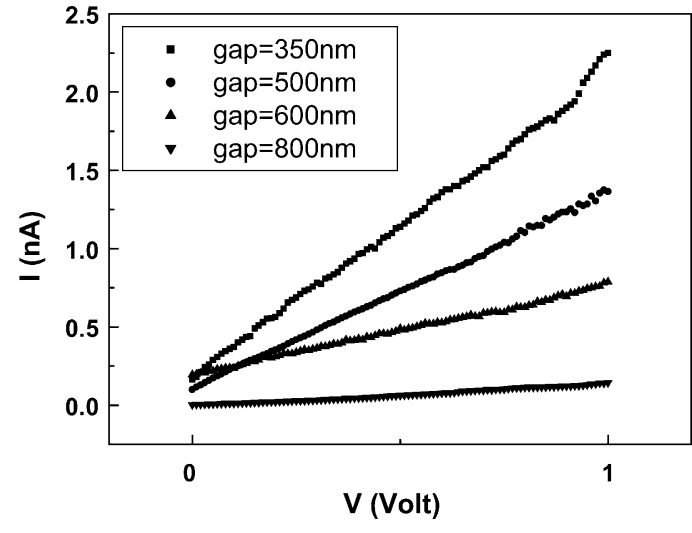

Fig. 7. Effects of nanogap distance on $I-V$ curve of self-assembly multilayer gold nanoparticles. The scanning rate is $10 \mathrm{mV} / \mathrm{s}$.

Table 2

Comparison between competing techniques

\begin{tabular}{llll}
\hline & $\begin{array}{l}\text { Silver } \\
\text { enhancement }\end{array}$ & $\begin{array}{l}\text { Lowest target DNA } \\
\text { concentration }\end{array}$ & $\begin{array}{l}\text { Standard CMOS } \\
\text { process }\end{array}$ \\
\hline$[4]$ & Yes & $500 \mathrm{fM}$ & No \\
{$[11]$} & Yes & $0.2 \mathrm{nM}$ & No \\
{$[12]$} & Yes & $1 \mathrm{pM}$ & No \\
Present study & No & $1 \mathrm{nM}$ & Yes \\
\hline
\end{tabular}

ticle increases to a value of $10 \mathrm{nA}$ at an applied voltage of $1 \mathrm{~V}$ (Fig. 6(b)). When stressing the bias voltage from 0 to $1 \mathrm{~V}$, current continuity is observed. It is noted that the concentration of target oligonucleotide in the sample solution is $1 \mathrm{nM}$.

The effect of nanogap distance on the $I-V$ curve for multilayer gold nanoparticles is also investigated. Four different distances of nanogap are used in the biochip. They are 350, 500,600 , and $800 \mathrm{~nm}$, respectively. Fig. 7 shows the electrical detection results of gold nanoparticle multilayer in nanogaps of width $350,500,600$, and $800 \mathrm{~nm}$, respectively. After gold nanoparticle multilayer is immobilized in the gap, the gap distance has significant effect on the $I-V$ curve through microelectrodes. The electrical conductivity through microelectrodes for multilayer gold nanoparticles decreases with the increase in gap distance.

\section{Conclusion}

An array-based CMOS biochip is designed and developed to replace the conventional DNA microarray for DNA identification by electrical detection. A novel technique using self-assembly gold nanoparticle multilayer to conduct and amplify electrical signal is presented. The electric current passing through gold nanoparticle multilayer, which is formed by complementary target oligonucleotide strands, exceeds that of gold nanoparticle monolayer by three orders of magnitude. The magnitude of electrical signal is primarily related to the density of gold nanoparticles immobi- lized in the gap and also the gap distance between the two electrodes.

The present CMOS biochip, which is fabricated by TSMC $0.35 \mu \mathrm{m}$ 1P4M CMOS process combined with post-CMOS micromachining processes, is of high yield rate for mass production. The recent competing techniques using electrical detection method are compared in Table 2 .

\section{Acknowledgements}

The authors deeply appreciate Chip Implementation Center (CIC) for providing all the design tools, TSMC for fabrication, and equipment for post-micromachining by NSC MEMS Research Center, Taiwan. The work in this study could not be achieved without their support.

\section{References}

[1] T.A. Taton, C.A. Mirkin, R.L. Letsinger, Scanometric DNA array detection with nanoparticle probes, Science 289 (2000) 1757-1760.

[2] T.A. Taton, R.C. Mucic, C.A. Mirkin, R.L. Letsinger, The DNAmediated formation of supramolecular mono- and multilayered nanoparticle structures, J. Am. Chem. Soc. 122 (2000) 6305-6306.

[3] T.A. Taton, G. Lu, C.A. Mirkin, Two-color labeling of oligonucleotide arrays via size-selective scattering of nanoparticle probes, J. Am. Chem. Soc. 123 (2001) 5164-5165.

[4] S.J. Park, T.A. Taton, C.A. Mirkin, Array-based electrical detection of DNA with nanoparticle probes, Science 295 (2002) 1503-1506.

[5] M. Lu, X.H. Li, B.Z. Yu, H.L. Li, Electrochemical behavior of Au colloidal electrode through layer-by-layer self-assembly, J. Colloid Interf. Sci. 248 (2002) 376-382.

[6] C.Y. Tsai, Y.H. Tsai, C.C. Pun, B. Chan, T.Y. Luh, C.C. Chen, F.H. Ko, P.J. Chen, P.H. Chen, Electrical detection of DNA hybridization with multilayer gold nanoparticles between nanogap electrodes, Microsyst. Technol. 11 (2005) 91-96.

[7] P. Caillat, D. David, M. Belleville, F. Clerc, C. Massit, F. RevolCavalier, P. Peltie, T. Livache, G. Bidan, A. Roget, E. Crapez, Biochips on CMOS: an active matrix address array for DNA analysis, Sens. Actuators B 61 (1999) 154-162.

[8] P. Swanson, R. Gelbart, E. Atlas, L. Yang, T. Grogan, W.F. Butler, D.E. Ackley, E. Sheldon, A fully multiplexed CMOS biochip for DNA analysis, Sens. Actuators B 64 (2000) 22-30.

[9] J.M. Yang, J. Bell, Y. Huang, M. Tirado, D. Thomas, A.H. Forster, R.W. Haigis, P.D. Swanson, R.B. Wallace, B. Martinsons, M. Krihak, An integrated, stacked microlaboratory for biological agent detection with DNA and immunoassays, Biosens. Bioelectron. 17 (2002) 605-618.

[10] P.E. Lobert, D. Bourgeois, R. Pampin, A. Akheyar, L.M. Hagelsieb, D. Flandre, J. Remacle, Immobilization of DNA on CMOS compatible materials, Sens. Actuators B 92 (2003) 90-97.

[11] L. Moreno-Hagelsieb, P.E. Lobert, R. Pampin, D. Bourgeois, J. Remacle, D. Flandre, Sensitive DNA electrical detection based on interdigitated $\mathrm{Al} / \mathrm{Al}_{2} \mathrm{O}_{3}$ microelectrodes, Sens. Actuators B 98 (2004) 269-274.

[12] J. Li, M. Xue, Z. Lu, Z.K. Zhang, C.G. Feng, M.S. Chan, A highdensity conduction-based micro-DNA identification array fabricated with a CMOS compatible process, IEEE Trans. Electron Dev. 50 (2003) 2165-2170.

[13] M.A. Hayat, Colloidal Gold: Principles, Methods and Applications, vols. 1/2, Academic Press, San Diego, CA, 1989. 


\section{Biographies}

Yi-Ting Cheng received his BSc degree in mechanical engineering from the National Taiwan University in 2001 and is currently pursuing the $\mathrm{PhD}$ degree from the Nation Taiwan University in Taipei, Taiwan, ROC. His main research interests include MEMS fabrication, CMOS-MEMS design, biochip for DNA detection, and microfluid system.

Ching-Chin Pun received the MSc degree in mechanical engineering from National Taiwan University, Taipei, Taiwan, ROC, in 2003. His research interests include BioMEMS fabrication, CMOS-MEMS design, system integration, and biomedical devices.

Chien-Ying Tsai was born in Yunlin, Taiwan, in 1972. He received his $\mathrm{PhD}$ degree from Taiwan University in 2004. His doctoral research focused on the determination of electricity of gold nanoparticles using DNA hybridization. In 2004, he worked as a postdoctoral research fellow at the Institute of Chemistry, Academia Sinica, Taiwan. His research interests include MEMS with emphasis on the mechanical properties of thin films using micromachined structures, CMOS process and self-assembly gold nanoparticle structure.

Ping-Hei Chen received his BSc degree from National Taiwan University in 1980, and $\mathrm{PhD}$ degree from the University of Minnesota in 1988. After graduation, he joined the Department of Mechanical Engineering of National Taiwan University as an associate professor. He was promoted to a full professor in 1996. He had served as the Chairman of the Department of Mechanical Engineering in National Taiwan University from 1998 to 2001. His research areas include micro-thermal-fluid systems, Lab-on-achip for DNA detection, Nanofluid, MEMS fabrication technology, and cooling devices for electronic equipment. 